\title{
Research on the Dilemma of Speculation in Chinese Stock Market Based on Game Theory
}

\author{
Dexiao Ren \\ School of Government, Beijing Normal University, Beijing, China \\ Email: dx0703@163.com
}

Received 23 June 2016; accepted 29 July 2016; published 1 August 2016

Copyright (C) 2016 by author and Scientific Research Publishing Inc. This work is licensed under the Creative Commons Attribution International License (CC BY). http://creativecommons.org/licenses/by/4.0/

(c) (i) Open Access

\begin{abstract}
In this paper, the Chinese stock market is analyzed from the perspective of participating in the game. Under the interaction of the participants, the model forms two stable equilibriums. The ideal equilibrium is that most people tend to invest in real investment rather than speculation. Speculation is not the mainstream of the market, however, most people choose to follow the trend of speculation, and the entire stock market speculation prevails, that is also a possible equilibrium. This paper argues that the current situation of Chinese stock market speculation is in a non-ideal equilibrium, which most people choose to speculate, and the overall market environment is inclined to choose speculation, and participants continue to choose speculation, and market as a whole environment speculation prevails. As long as the belief of participant to the market environment does not change, they will not change their economic decision and the market environment will still trend to speculation, which is why the guide of regulatory department does not work and speculation in the stock market continues the root cause.
\end{abstract}

\section{Keywords}

Stock Market Speculation, Stock Market Investment, Game Equilibrium

\section{Introduction}

There has been more than 20 years since the establishments of China's stock market, during this period, although regulators continue to conduct a variety of attempts to guide investors to make rational investment, combat control of the market, insider trading and other acts. But the speculation such as the concept hype, the shell speculation, the bull market of policy etc. in Chinese stock market is still prevalent. In 2015 the Chinese stock market staged a huge 
shock was to make people feel deeply the stock market speculation. This article will analyze the dilemma of China's stock market speculation from the perspective of the participants in the game of stock trading. This article will discuss the relationship between the economic decisions and the whole environment of the stock market, as well as the reason of the stock market speculation.

\section{Literature Review}

The study on the phenomenon and reason of Chinese stock market speculation has been started since the date of the establishment of Shanghai and Shenzhen stock markets. On the stock market "game", "Bo dumb" controversy has been continued. Early in December 1996, "people’s Daily" special review article mentioned the stock market to the five problems: large institutional market manipulation; bank illegal funds into the market; illegal securities institutions overdraft; news media adding fuel to the flames; misleading mistakenly believe investors to follow suit. In June 2000, a title for the shady Fund-Fund Behavior Research Report Analysis, the article is about the Chinese stock market argument become fuse, Wu Jinglian, Li Yining, Xiao Zhuoji etc. among scholars is heated debate broke out. The controversy about the stock market, "gamble" later slowly in silence, but controversy about the Chinese stock market excessive speculation still continues, Lv Jianglin and Zeng Peng [1] comprehensive analysis of the indicators, China's stock market dividend payout ratio and dividend yield, the exchange rate, wealth effect, Tobin's q effect etc., that Chinese stock market is still speculation driven in the stock market.

Accompanied with the stock market gambling dispute are reasons for speculation on the stock market research, many scholars analyzed and summarized. The reason can be roughly divided into three categories. The first view is that because the stock cannot provide enough dividends benefit and as a guarantee of the rights and interests of the shareholders, such as Hao Jilun [2] consider that the direct reason of Chinese stock market speculation is the low return rate and the distortion of stock right structure, as a return of the securities and the securities of the stock can not reflect the fundamental characteristics of these two aspects. The second type of view is that the fundamental reason for the stock market speculation is that the Chinese government to plan to operate the market, resulting in the market presents the characteristics of the policy market, Wen Binzhou [3] [4] consider the underlying reason for the Chinese stock market speculation is that the government operates the market in a planned system, government habits through a series of good or bad policy about the direction of the volatility of the stock index, in essence, there is no difference with the government to use administrative means to regulate and control the real economy. Yi Xianrong [5], Wang Guogang [6] and other scholars hold similar views. The third kind of view thinks that the reason of Chinese stock market speculation is comprehensive, Huang Xiangzhen [7] agree that China's stock market cannot avoid speculation, First, because China's stock market system is not perfect, the second reason is the blue chip stocks cannot give investors a satisfactory return, the third reason is the operation of the stock market funds more speculative, speculation in the atmosphere led to the small and medium retail to join the market speculation.

This paper believes that the analysis of Chinese scholars in the past, the stock market speculation on the direct and fundamental reasons for more discussion, but it is difficult to explain why more than 20 years of stock market speculation prevailed in the situation cannot be reversed. And previous scholars more concerned about the stock market participants' external factors', such as the government, the system, the listing Corporation equity structure, etc. The interactive relationship between stock market participants and the overall environment of the stock market is seldom considered. With the development of China's society, the idea of governance of Chinese government has changed gradually. The basic system of the stock market has been perfected, but the phenomenon of excessive speculation in the stock market has not been reduced. By the end of 2014, Wu Jinglian [8] once again put forward the "stock market casino theory" that the Chinese stock market is not only like a casino, but also a no rules of the casino. As if to prove Professor Wu's point of view, in half a year later, China's stock market appeared a huge shock, speculative market characteristics of take in everything in a glance. Therefore, this article will use the method of game theory to think again about the stock market speculation.

At present, using game theory to study the stock market speculation has been a certain amount of literature. Wang Jiankun [9] analyzed the game between Chinese stock market exchange, market maker, retail investors and government based on game theory. Qiao Jianming [10] analyzed the operation process and evolution trend of the "policy market" characteristics of Chinese stock market. Hao Xuguang [11] analyzed the interaction between the government and the medium and small investors in two cases of market overheating and market 
downturn. Liu Wenchen [12] using the method of game theory analysis of the government, the dealer, retail and China stock market in the four village people involved in human interaction, in the government and the banker's game, it can reduce the probability of the dealer violation to increase the punishment for insider trading, stock market manipulation and through the establishment of specialized agencies, reduce the cost of the review of illegal activities. But few scholars will focus in the use of game theory to discuss the predicament of speculation in the Chinese stock market, also scholars in the past when we use game theory to analyze stock market participant interaction, usually in divided into government, transaction, the dealer, village, retail and other, in specific game process in each other to start the game. This paper argues that this division is not conducive to the analysis of the overall balance of the stock market. In the stock market, participants are divided into the village, and small and medium-sized retail dealer, which Cannot show a tendency to be involved in the face of speculation or investment, and rational government hopes to maintain the order of the capital market, and promote the stock market to mature, and the subjective choice of the strategy will be the regulation or punishment. So how a single transaction in the Chinese stock market is influenced by the choice of speculation or real investment decisions on the overall market? After more than 20 years of development, China's stock market is still filled with a strong speculative atmosphere, it seems that this state has become a kind of non break the balance, why it form this situation? This article will analyze the above issues from the perspective of the game equilibrium of the participants in the stock market, in order to clarify the dilemma of China's stock market speculation, and to provide some references for the long-term and fundamental governance of China's stock market.

In this paper, the main content is: the first part is introduction, the second part is the total number of literature, the third part for investment and speculative static model analysis, including the model hypothesis and establishment, equilibrium analysis, the fourth part is a comparative static analysis of the model. The last is the conclusion.

\section{Static Game Model of Investment and Speculation}

This part focuses on the behavior of stock market participants other than government regulators. Each participant can no longer distinguish between the village and the small and medium-sized retail dealer, but directly according to the different tendency in partition strategy. In order to explore the overall market equilibrium of stock market participants, this paper constructs a static game model based on all participants.

\subsection{Participant ${ }^{1}$}

The participant in the model is the natural person or organization to put capital into the stock market. In order to maximize the individual interests, the stock market participants will gather information through a variety of channels to collect information, and then according to the market environment information to make decisions in line with the interests of individuals. And the stock market is composed of a number of participants, but usually each of the participants themselves and will not take into account how their own decisions impact on the overall market environment. Strategy is preferred as the basis of this distinction; define the type of participant as $\theta$. The $\theta$ value is higher, more inclined to invest but not speculative; on the contrary, people involved in the lower value, speculation is higher, the lower the tendency of real investment.

According to the definition of $\theta$ value, $\theta$ range is $(-\infty,+\infty)$, on this interval, distribution function and density function of $\theta$ respectively are $H(\theta), h(\theta)$. A person who is totally inclined to speculate or invest is only a small part of the population, most people are in the middle of the range, It is generally believed that the participants who hold investment and speculative tendencies are in a normal distribution in the population. So $H(\theta)$ is normal distribution function.

\subsection{Strategy Set}

In the model, there are two kinds of people who are involved in economic decision-making.

\subsubsection{Real Investment Strategy}

This strategy means that the participants will carry out a comprehensive analysis and research to the fundamentals of the company, market environment, and stock market performance before they make stock trades and they

${ }^{1}$ If no special instructions, the stock market participant in particular to stock market investors, because in the game model, strategy set contains speculative investment, two strategies, using the term investors easily misunderstanding, therefore takes the person refers to the stock market investors. 
are willing to engage in the management and supervision of enterprises and earn dividends.

\subsubsection{Speculation Strategy}

Speculation strategy prefers various types of topics and concepts of speculation, ignoring the basic situation of enterprises, through the one-sided or informal channel news stock trading.

\subsection{Stock Market Environment}

In this paper, the stock market environment refers to the market speculation or investment climate, which is formed by the participant interactions. The model is set in two states, mature and immature. In the mature stock market, speculation is not recognized, and shareholders to participate in the business sense of supervision and management, the interests of small shareholders are more secure. Conversely, in the immature stock market, speculation prevails, the shareholders' participation in the corporate supervision and management is weak, the interests of small shareholders are difficult to guarantee.

Because the stock market is composed of many participants, the environment of the stock market depends to a certain extent on the participant's strategy choice behavior. At the same time, the environment of the stock market will affect the participants to make economic decisions in turn. To have a higher propensity to invest in people and mature stock market is clearly more conducive to obtain a higher return on investment, in the immature stock market, enterprise behavior by from weaker shareholder supervision, make it difficult to guarantee the interests of shareholders, such entities in the mature market to support attitude or behavior actually for the market to mature. And with high speculative tendencies in people, immature stock market will be more conducive to the gain, because mature market, speculation is often made difficult by the recognition that insider trading is extremely limited, through frequent trading access to spread more difficult, such entities does not support or its behavior in fact not conducive to market maturity.

In this paper, the $x$ representative proportion of participants to support the market mature or its behavior is conducive to the market to mature, obviously $0 \leq x \leq 1$, so $1-x$ representative proportion of participants to not support the market mature or its behavior isn't conducive to the market to mature. And whether the stock market is mature or not, it is related to the proportion of participants who are involved in the market to mature. Assuming that probability that market will eventually be able to mature is $\pi, \pi=\pi(x)$ is increasing function of $x$, that is $\pi^{\prime}=\mathrm{d} \pi / \mathrm{d} x>0$. Consider the two extreme cases, $x=0$ means that all in per capita do not want to see the market matured, then a mature market is unlikely, therefore make $\pi(0)=0 ; x=1$ means the opposite, then possibility of mature market greatly, and therefore make $\pi(1)=1$.

From the above analysis at the same time, we can have the following relationship:

$$
x=1-H(\theta) .
$$

$H(\theta)$ is proportion of participants whose type value less than $\theta$, function curve of Equation (1) is as Figure 1. Point $(x, \theta)$ on the curve represent when the participant whose type value greater than $\theta$ tend to investment, the proportion of participant who is support mature market or whose behavior is conducive to the market to mature is $x$; or when the proportion of participant who is support mature market or whose behavior is conducive to the market to mature is $x$, the participant whose type value greater than $\theta$ tend to investment.

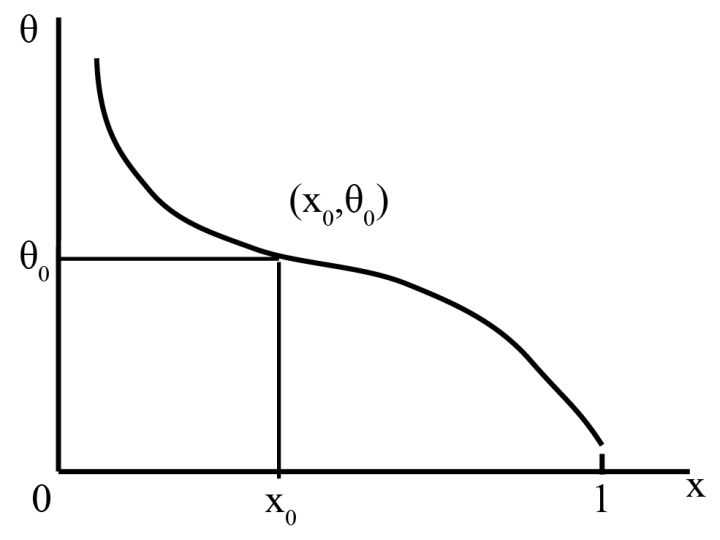

Figure 1. Investment tendency curve. 


\subsection{Payoff of Participants}

Market participant $\theta$ has two strategies; market environment also has two possible results. Therefore, market participants have a total of four forms of payoffs. $V$ and $S$ express the strategies of investment and speculation respectively, and $M$ and $N$ represent the mature and immature stock markets respectively.

For the investment strategy, the payoffs of the two kinds of market conditions are:

$$
V^{M}=v(\theta), V^{N}=\left(1-\lambda_{1}\right) v(\theta) .
$$

The type of people involved in the value of $\theta$ represents its investment or speculation, for those who have a high propensity to invest, often look for more market opportunities, enhance the ability to find and grasp. Therefore, it can be considered to have a higher propensity to invest in people, its investment capacity is also stronger, the income gained through investments higher, so $\mathrm{d} v / \mathrm{d} \theta>0$. In addition, $\lambda_{1}$ represents an immature market environment led to the proportion of investment strategy revenue decline, so $0 \leq \lambda_{1}<1$, when $\lambda_{1}=0$, Means that the investment strategy is not due to the impact of the market is not mature.

For the speculative tendency of the strategy, the two kinds of market conditions are:

$$
S^{M}=\left(1-\lambda_{2}\right) s(\theta), S^{N}=s(\theta) .
$$

Similarly, for smaller theta value has higher tendency of people to participate in speculation, usually more willing to use the market speculation and a variety of non-normal channel news, frequent low buy high sell speculation, and therefore its ability to speculate and income from speculate, so $\mathrm{ds} / \mathrm{d} \theta<0 . \lambda_{2}$ represents a mature market environment led to the decline in the proportion of speculative strategy revenue, so $0 \leq \lambda_{2}<1$, when $\lambda_{2}=0$, Means that the return on the market is not mature because of the impact of the market maturity.

From this, the stock market participant's payoffs structure is as follows (Figure 2).

Focus of this paper is to analyze the differences between different strategies for payment, in this set $\Delta$ is the difference between investment and speculation strategy of payment, so:

$$
\begin{aligned}
\Delta(x, \theta) & =\pi V^{M}+(1-\pi) V^{N}-\left[\pi S^{M}+(1-\pi) S^{N}\right] \\
& =\pi v(\theta)+(1-\pi)\left(1-\lambda_{1}\right) v(\theta)-\pi\left(1-\lambda_{2}\right) s(\theta)+(1-\pi) s(\theta) \\
& =\left(1-\lambda_{1}+\pi \lambda_{1}\right) v(\theta)+\left(\pi \lambda_{2}-1\right) s(\theta) .
\end{aligned}
$$

When $\Delta(x, \theta)=0$, we could get a Implicit function about $x$ and $\theta$, every point $\theta^{*}(x)=(x, \theta)$ on the function curve means that to the participate whose type is $\theta$, there will be a support for the market is mature or its behavior is conducive to the market to mature proportion $x$ of the population to makes same payoffs from the choice of investment strategy and speculation strategy. And for the point beyond the function curve, those type value $\theta<\theta^{*}(x)$ choose speculation strategy and don't support or their behaviors have no help to the market being mature. And those type value $\theta>\theta^{*}(x)$ choose investment strategy and support or their behaviors have help to the market being mature. So,

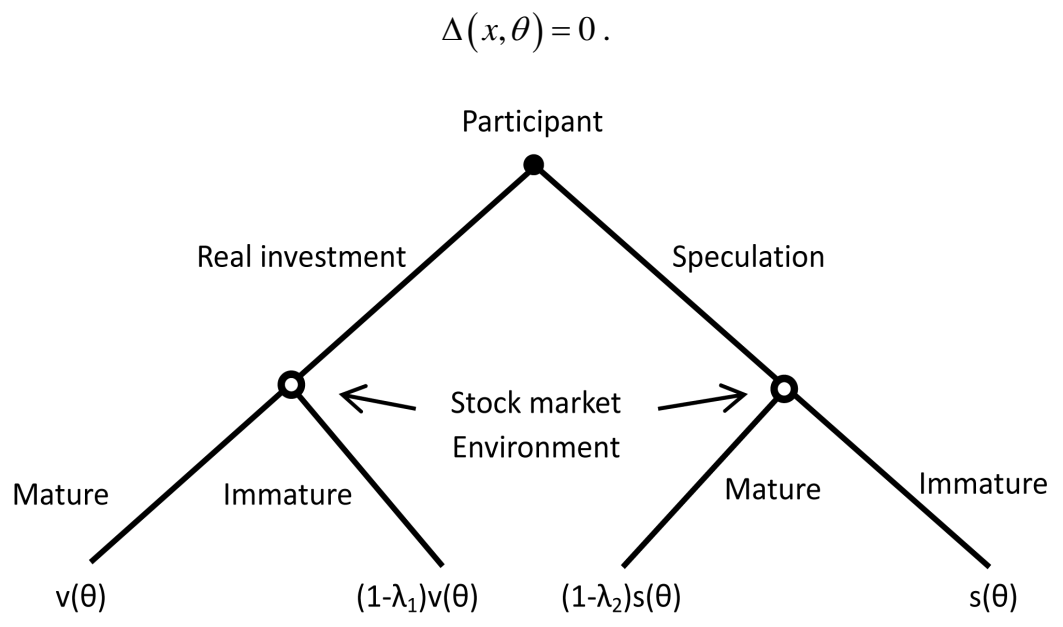

Figure 2. Participant strategy payoff structure. 
The above curve can be called the investment and speculation of no difference curve or conversion line. Because,

$$
\mathrm{d} \theta / \mathrm{d} x=-\frac{\Delta_{x}(x, \theta)}{\Delta_{\theta}(x, \theta)}=-\frac{\pi^{\prime} \lambda_{1} v(\theta)+\pi^{\prime} \lambda_{2} s(\theta)}{\left(1-\lambda_{1}+\pi \lambda_{1}\right) \mathrm{d} v / \mathrm{d} \theta+\left(\pi \lambda_{2}-1\right) \mathrm{d} s / \mathrm{d} \theta}<0 .
$$

Monotone decreasing curve, the concrete manifestation is with $X$ as the horizontal axis, with the longitudinal axis of the coordinate system for $\theta$, tilt under the curve to the right (as shown in Figure 3).

\subsection{Equilibrium Analysis}

According to Equation (1) and Equation (2), the $x$ value of the equilibrium can be got,

$$
x=1-H\left[\theta^{*}(x)\right] .
$$

Corollary: there is at least one solution of Equation (4). Assuming the solution is $x^{*}\left(0<x^{*}<1\right)$, If at this point to meet,

$$
-h\left(\theta^{*}\right) \mathrm{d} \theta\left(x^{*}\right) / \mathrm{d} x>1 .
$$

Equation (4) has at least two other solutions, in the two solutions, one is greater than $x^{*}$, one is less than $x^{*}$; If for all $x$, the inequality (5) does not hold, the solution or the equilibrium is unique.

Prove: Constructing auxiliary function $\varnothing(x)$, make $\varnothing(x)=1-H\left[\theta^{*}(x)\right]-x$.

So $\varnothing(0) \geq 0, \varnothing(1) \leq 0$, and because $\varnothing(x)$ is continuous function, According to the zero point theorem, there will have at least one point in $(0,1)$ make $\varnothing(x)=0$.

Because $x^{*}\left(0<x^{*}<1\right)$ is a solution of the equation, $\varnothing\left(x^{*}\right)=0$.

If the inequality (5) is set up, derivative of $\varnothing(x)$ at $x^{*}$ is $\varnothing\left(x^{*}\right)^{\prime}=-h\left(\theta^{*}\right) \mathrm{d} \theta\left(x^{*}\right) / \mathrm{d} x-1>0$, so $\varnothing(x)$ at least exists two other solutions on [0,1]. The three solutions are shown in Figure 4.

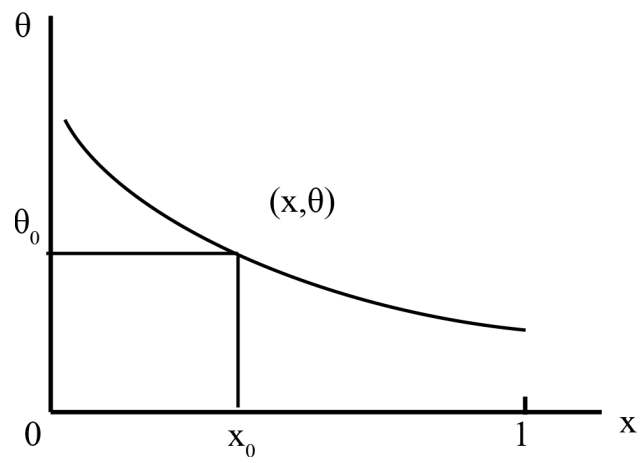

Figure 3. The payoff of the strategy of speculation and speculation no difference curve/conversion curve.

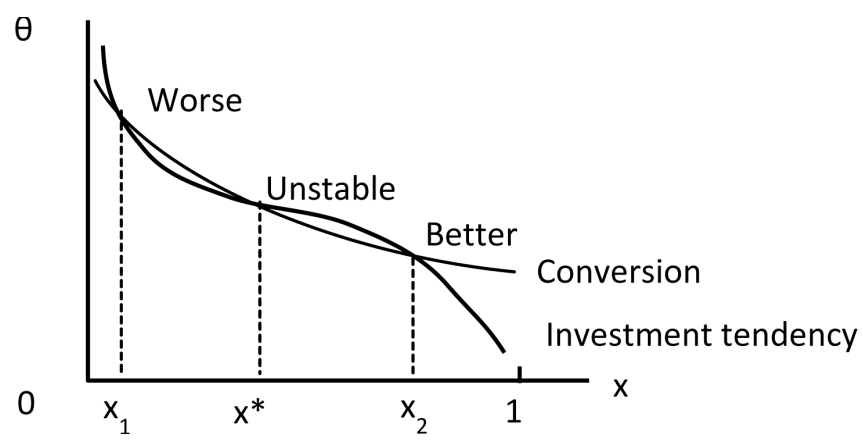

Figure 4. Equilibrium analysis: good balance and poor balance. 
If for all $x^{*}$, the inequality (5) does not hold, then the solution is unique.

Another meaning of inequality (5) were established when the $X$ in $X^{*}$ accidental fluctuation on $X, H(\theta)$ correspondingly greater than changes in the $X$. In this case, the $x^{*}$ equilibrium is not a stable equilibrium. Because the fluctuation of $X$ can make the whole environment of the stock market have more changes, which affect the judgment of the participants' beliefs about other subjects' behavior. Ultimately make it change its own economic decision-making, and the participant who changed his decision will not spontaneously change back to its original economic decisions.

Compared with the $x^{*}$ in Figure 4, $X_{2}$ and $X_{1}$ are two stable equilibrium, But it is obvious that there are obvious differences between the two equilibrium in the stock market and the behavior tendency of the main body in the stock market and the maturity of the market: $X_{2}$ balance will be more involved in the choice of investment, the stock market is also the possibility of the final maturity of the larger, $X_{1}$ at the opposite.

Based on the above analysis, the conclusion that can be drawn from the model is that, there may be two equilibrium states in the stock market. A good balance is the market participant to rational, real investment, the market will eventually become mature, but there may also be a poor balance of the formation. Stock market participants tend to make speculate strategy, and the formation of a "immature stock market, speculation in the interests of individuals" beliefs, Under the influence of this kind of belief, the participant is more inclined to take the speculation strategy, the investment faces the great barrier, the stock market is stable in the immature state. In such a stable state, the market is usually not spontaneous change, only when there is enough to participate in the market environment is expected to be a great change. For personal interests consider the need to change the personal strategy when the whole market is likely to form a better balance. But this change is almost impossible in a very short time, through a small part of the effort to complete. This also explains in the Chinese stock market, why a management constantly strives to standardize the market, but on the other hand, the stock market is still speculation, real investment or investment philosophy cannot always be the mainstream of the market strategy.

\section{Comparative Static Analysis}

If we make the stock market and other stakeholders being into the scope of consideration, their impact on the model in the short term will be the main performance of the two curves to move up and down. For example, the government introduced more stringent provisions of the stock market information disclosure; Increase the crackdown on insider trading, which will enhance the people involved in the stock market to mature awareness and confidence to move the transfer curve downward. Figure 5(a) shows the downward movement of the transition curve, two new stable equilibrium points are $x_{1 a}^{\prime}, \quad x_{2 a}^{\prime}$. The new equilibrium point has higher $x$ value compared to the original equilibrium point, which means that there are more people to support the market mature or their behavior is conducive to the market to mature, The possibility of more mature market, and participate in the human type is low, Means that the original investment tends to have a slightly lower investment at this time is also willing to invest, the downward shift of the curve has brought a better balance of results. Similarly, if the government to take a more comprehensive protection of the rights and interests of small shareholders, listed

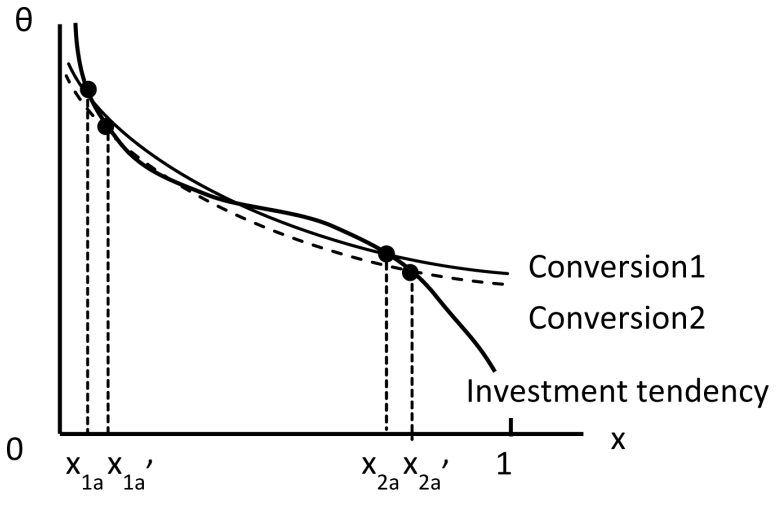

(a)

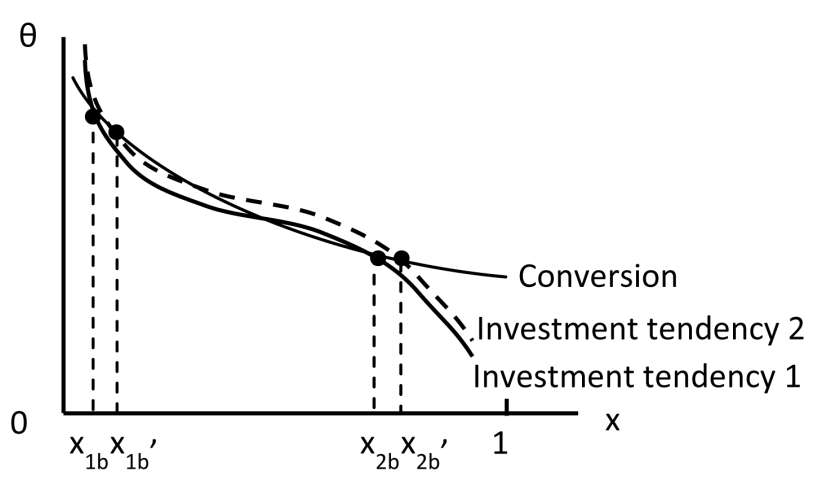

(b)

Figure 5. (a) Conversion line down. (b) Investment tendency curve up. 
companies more respect for shareholders to receive dividends, the right to participate in business management, will also be involved in the investment to produce greater New gravity, to promote the investment tendency curve up.

Figure 5(b) shows the upward movement of the propensity to invest; there exists two stable equilibrium points of $x_{1 b}^{\prime}$ and $x_{2 b}^{\prime}$ than their original equilibrium point also has a higher $x$ value and lower $\theta$ value. The upward trend of the investment tendency curve also brings a better-balanced result.

The behavior of other stakeholders can affect the investment tendency curve and the transfer line, to a certain extent, the equilibrium state can be better or worse, But only in the belief of the stock market participants greater changes, the balance will be a fundamental transformation, this may depend on the overall concept change of the participant, For example, the stock market investors to invest in the idea of a more mature, More institutional investors to enter the stock market to bring the proportion of people involved in the optimization, etc.

\section{Conclusions}

This paper analyzes the situation of the Chinese stock market speculation from the perspective of participant game. Due to the interaction of the market participants' economic behavior, the model has the potential to form two stable equilibrium states. The ideal equilibrium is that most market participants tend to invest more in real investment rather than speculation, and speculation is not the mainstream of the market. But there is also another kind of non ideal equilibrium, at this time most people choose to follow the trend of speculation, and the stock market speculation prevails.

In the stock market, it is difficult for the individual participants to impact the stock market environment, but the decision of the most investors, speculation or real investment, will have a different impact on the stock market environment. So the economic decision of the stock market participants and stock market environment becomes closely related. The participants are affected by the market environment, and in turn affect the market environment, and work together to influence the overall investment environment. In this sense, the status of the Chinese stock market speculation is in a non-ideal equilibrium that "most participants choose the speculation, overall market environment tends to speculation, participants choose the speculation, and the overall market environment continues to favor speculation”. As long as the belief of participant to the market environment does not change, they will not change their economic decision and the market environment will still trend to speculation, which is why the guide of regulatory department does not work and speculation in the stock market continues the root cause.

\section{References}

[1] Lv, J.L. and Zeng, P. (2012) Research on the Nature of "speculation Leading” in China's Stock Market. Statistics and Decision Making, No. 8, 157-160.

[2] Hao, J.L. (1997) An Institutional Analysis of Excessive Speculation in the Stock Market of China. Science of Finance and Economics, No. 2, 7-10.

[3] Wen, B.Z. (1996) On Speculation and Investment in the Stock Market. Contemporary Economic Science, No. 4, 94-95.

[4] Wen, B.Z. and Yang, Y.Q. (2014) Administrative Control, Speculation and the Dilemma of Stock Market: A Review of China's Stock Market in 20 Years. Financial Theory Research, No. 4, 88-96.

[5] Yi, X.R. (2005) The Focus and Difficulty of China’s Stock Market Policy Reform in 2005. Chinese Finance, No. 2, 50-51.

[6] Wang, G.G. (1996) Theoretical Thinking on Some Issues of China’s Capital Market—On “Nine Five” Capital Market Orientation and Dynamic. Finance and Trade Economics, No. 4, 13-19.

[7] Huang, X.Z. (2006) On Value Investment and Speculation in Chinese Stock Market: On the Combination of Theory and Practice of "Securities Investment”. Journal of Fujian Normal University, No. 3, 91-95.

[8] Wu, J.L. (2000) Talk about the Stock Market for Ten Years. Far East Press, Shanghai.

[9] Wang, J.K. (2000) The Game Analysis of Chinese Stock Market. Finance and Trade Economics, No. 2, 54-58.

[10] Qiao, G.M. (2004) The Game Analysis of “Policy Market” in Chinese Stock Market. Economic Science, No. 2, 65-73.

[11] Hao, X.G., Zheng, L.J., et al. (2012) The Game Analysis of Securities Market Regulators and Small Investors. The Theory and Practice of Finance and Economics, No. 9, 41-44.

[12] Liu, W.C. and Zhu, J.M. (2012) Game Analysis of Stock Market Investment. Journal of Central University of Finance and Economics, No. 5, 44-47. 


\section{Submit or recommend next manuscript to SCIRP and we will provide best service for you:}

Accepting pre-submission inquiries through Email, Facebook, LinkedIn, Twitter, etc.

A wide selection of journals (inclusive of 9 subjects, more than 200 journals)

Providing 24-hour high-quality service

User-friendly online submission system

Fair and swift peer-review system

Efficient typesetting and proofreading procedure

Display of the result of downloads and visits, as well as the number of cited articles

Maximum dissemination of your research work

Submit your manuscript at: http://papersubmission.scirp.org/ 